\title{
Oblique Lateral Interbody Fusion Combined With Different Internal Fixations in The Treatment of Degenerative Lumbar Spine Disease: A Finite Element Analysis
}

ShuYi Zhang

Affiliated Hospital of Chengde Medical College

Zhengpeng Liu

Affiliated Hospital of Chengde Medical College

Chenshui Lu

Fuzhou University

Li Zhao

Nanfang Hospital

Chao Feng

Chengde Central Hospital

Yahui Wang

Affiliated Hospital of Chengde Medical College

Yilong Zhang ( $\triangle$ 952714988@qq.com )

Affiliated Hospital of Chengde Medical College

Research Article

Keywords: oblique lumbar interbody fusion, finite element, bilateral pedicle screw

Posted Date: November 10th, 2021

DOI: https://doi.org/10.21203/rs.3.rs-991669/v1

License: (c) (i) This work is licensed under a Creative Commons Attribution 4.0 International License.

Read Full License 


\section{Abstract}

Little is known about the biomechanical performance of various internal fixations in oblique lumbar interbody fusion (OLIF). In this study, finite element (FE) analysis was used to describe the biomechanical findings of various different internal fixations to compare and explore the stability of each fixation.

\section{METHODS:}

Six validated FE models of the L3-S1 segment were reconstructed from computed tomography images, including (1) an intact model, (2)a stand-alone (SA) model with no instrument (3) a unilateral pedicle screw model (UPS), (4) a unilateral pedicle screw contralateral translaminar facet screw model (UPSCTLFS), (5) a bilateral pedicle screw (BPS) model, and (6) a cortical bone trajectory screw (CBT).Threedimensional model was performed by computed tomography data, and $150 \mathrm{~N}$ static force and $10 \mathrm{~N} . \mathrm{m}$ moments in different directions were applied to the models to analyze the validation of the models in comparison with previous studies. Models of the OLIF cage was created with three-dimensional scanning to improve the accuracy of the FE analysis. Range of motion (ROM) of the surgical segment stresses, stress of the cage, and stress of fixation were evaluated in the different models.

\section{RESULTS:}

ROM increased from least to greatest as follows: BPS, UPS-CTFS, CBT, UPS, SA. The SA group had the greatest ROM and the SA group had the greatest ROM and stresses on the CAGE. The ROM of the BPS and UPS-CTFS was not significantly different for all motion loadings. Compared with the other three models, the BPS model had lower stresses in internal fixation for all loading conditions and the CBT screw internal fixation had the highest stresses for different loads Compared with the other groups.

\section{CONCLUSIONS}

The BPS model provided the best biomechanical stability for OLIF. The SA model was relatively less stable. The UPS-CFTS group reduced the ROM of the fusion segments, but the stresses on the internal fixation and CAGE were relatively higher in the UPS-CFTS Compared with the BPS group; and the CBT group had lower ROM in flexion and extension Compared with the BPS, but its ROM in rotation and lateral flexion was relatively higher. The stability of the CBT was poorer than that of the BPS and LPS-CTFS groups. The stress on the CAGE and internal fixation was greater in the CBT group.

\section{Introduction}

In 1911, Albee [1]and Hibbs [2]described a posterior lumbar interlaminar fusion treatment for thoracolumbar tuberculosis. Since that time, there has been a trend toward lumbar interbody fusion achieving similar surgical goals as the procedure described by Albee and Hibbs for cases of lumbar spinal instability. With the development of minimally invasive spinal fusion technology, various and less invasive fusion methods, such as minimally invasive transforaminal lumbar interbody fusion (MIS-TLIF), 
extreme lateral interbody fusion (XLIF), and oblique lumbar interbody fusion (OLIF), have been developed in recent decades[3].In 1997, Mayer [4] first described a minimally invasive anterior approach to the lumbar spine through retroperitoneal access at the L2-L5 level and transperitoneal access at the L5-S1 level. In 2012, Silvestre et al[5]. used a minimally invasive retroperitoneal anterior approach similar to Mayer's approach for anterior lumbar interbody fusion. This technique is referred to by Silvestre et al. as OLIF, which is an aorta-psoas approach. This novel fusion procedure has been immediately recognized and adopted by spinal surgeons worldwide[6, 7].

From the patient's left side, there is a clear corridor from the L2 to the L5 vertebra among skin, the psoas and the aorta that averages approximately $18 \mathrm{~mm}$. This corridor can be further expanded to an average of $26 \mathrm{~mm}$ by blunt dissection of muscle fiber and gap between fascia[6]. This corridor allows direct access to the diseased disc without opening the spinal canal and damaging the posterior muscles, ligaments and bony structures; at the same time the OLIF technique substantially increases the support strength of the fusion because sufficient disc tissue can be removed, and the fusion has a large contact area with the endplate and can be extended to the sides over the dense bone protuberance surrounding the vertebral body $[6,8,9]$. Disruption of the psoas muscle and lumbosacral plexus can also be avoided by OLIF[10-12]. However, there are still some controversies about OLIF like whether to add internal fixation, what type of fixation is needed; current reported surgical methods are SA and OLIF+UPS, but OLIF+BPS or CBT is rarely reported, $[6,13,14]$ and few studies have investigated the biomechanical properties of OLIF with various fixation options.

FE analysis and cadaver experimental studies are complementary techniques for characterizing the complex biomechanical properties of the lumbar vertebrae $[15,16]$ Compared with cadaveric testing, $\mathrm{FE}$ method can simulate different internal fixations conveniently and allows the determination of many values (including internal stresses and strains). Therefore, it has been used in the spinal surgery to find solutions for different problems for decades. This study seeks solutions to the problem of FE homology or clarifies the suitability of new implants for clinical use, which can be determined by Finite Element Analysis (FEA) of the biomechanical differences between different device options for OLIF.

\section{Materials And Methods}

\subsection{Grouping}

Six FE models of the lumbar spine were created in this study. The FE models included (1) an intact model, (2) a stand-alone (SA) model with no instrument,(3) a unilateral pedicle screw model (UPS), (4) a unilateral pedicle screw contralateral translaminar facet screw model (UPS-CTLFS), (5) a bilateral pedicle screw (BPS) model, and (6) a cortical bone trajectory screw (CBT)

\subsection{Construction of the Intact Model}

A 38-year-old patient with degenerative lumbar spine disease was selected. 481 computed tomography images (Siemens 128 slice 64-row, SOMATOM Definition AS spiral CT, Germany) with a slice thickness of 
$0.625 \mathrm{~mm}$ were provided by the the Affiliated Hospital of Chengde Medical College. The computed tomography images were stored in Digital Imaging and Communications in Medicine format (DICOM). The collected raw data in the DICOM were imported into Mimics Research 21.0 (Materialise, Belgium) for three-dimensional (3D) reconstruction. Subsequently, the 3D model generated by Mimics was imported into Geomagic wrap 18 (reverse engineering software, USA); The noise and smoothing model were removed and cancellous bone and posterior structure were created and imported into SolidWorks 2020 (CAD software, Dassault Systemes, USA). Articular cartilage and intervertebral disc were created and the nucleus pulposus accounts for approximately $50 \%$ of the disc. The thickness of the cortical bone was 1 $\mathrm{mm}$ and the thickness of the vertebral endplate and cartilage endplate was set to 0.5 [15-19]. At the same time, the implant models [Table 1] were created, and the lumbar spine model was assembled. (In this experiment, the lumbar spine of patients with abnormal height of lumbar intervertebral space was not used and the intervertebral space of the model was not modified. Since the intervertebral space is indirectly elevated to the ideal height or position by cage after OLIF operation, the model was directly used here). The relevant implants were added to the patient's lumbar spine model in solidworks for Boolean operation. At the same time, the cartilage end plate, fiber ring and all nucleus pulposus needed to be removed in the OLIF operation were removed.

Table 1

Manufacturers and specifications of various different internal fixations

\begin{tabular}{|c|c|c|c|}
\hline Implants & Manufacturers & \multicolumn{2}{|c|}{ Specifications (Unit: mm) } \\
\hline \multirow[t]{2}{*}{ Pedicle screws } & \multirow[t]{2}{*}{ Weigao,Shandong } & $L=50$ & $D=4.5$ \\
\hline & & $L=45$ & $D=4.5$ \\
\hline cortical bone screw & LIBEIER,Beijing & $L=50$ & $W=4.5$ \\
\hline Facet screw & Weigao,Shandong & $L=44$ & $W=4.5$ \\
\hline \multirow[t]{2}{*}{ Cage } & Johnson \& Johnson(Ocrale) & $L=45$ & $\mathrm{H}=9$ \\
\hline & & \multicolumn{2}{|c|}{$0^{\circ}$} \\
\hline Rod & Weigao,Shandong & \multicolumn{2}{|c|}{$R=5.5$} \\
\hline \multicolumn{4}{|c|}{ In specification, L, D, W, R represents Length, Diameter, Width, and Radius respectively } \\
\hline
\end{tabular}

These models were imported into ANSYS Workbench 2020 R2 (ANSYS, Ltd., Canonsburg, Pennsylvania, USA) for pre-processing, and corresponding material parameters were set for each component [ Table 2] [16, 20-23]. Ligaments were simulated using springs subject to only pullout force [ Table 3] [24-27]. 
Table 2

Material properties of each part of the FE

\begin{tabular}{|lll|}
\hline Components/Materials & $\begin{array}{l}\text { elastic modulus } \\
\text { (MPA) }\end{array}$ & Poisson ratio \\
\hline cortical bone & 12000 & 0.3 \\
\hline cancellous bone & 100 & 0.2 \\
\hline posterior structures & 3500 & 0.25 \\
\hline anulus fibrosus & 4.2 & 0.45 \\
\hline vertebral endplate & 12000 & 0.3 \\
\hline cartilage endplate & 25 & 0.4 \\
\hline articular cartilage & 50 & 0.3 \\
\hline titanium alloy & 110000 & 0.3 \\
\hline Cage & 3600 & 0.3 \\
(polyetheretherketone,peek) & & 0.3 \\
\hline allogeneic bone & 3500 & 0.499 \\
\hline nucleus pulposus & 1 & \\
\hline
\end{tabular}

Table 3

FE parameters for each part of the ligament

\begin{tabular}{|lllll|}
\hline Major ligaments & $\begin{array}{l}\text { E } \\
(\mathrm{MPA})\end{array}$ & $\begin{array}{l}\mathrm{A} \\
\left(\mathrm{MM}^{2}\right)\end{array}$ & $\begin{array}{l}\mathrm{L} \\
(\mathrm{mm})\end{array}$ & $\begin{array}{l}\mathrm{K}=(\mathrm{A} \cdot \mathrm{E}) / \mathrm{L} \\
\left(\mathrm{kg} \cdot \mathrm{M}^{-2} \cdot \mathrm{S}^{-2}\right)\end{array}$ \\
\hline $\begin{array}{l}\text { Anterior longitudinal ligament } \\
\text { (ALL) }\end{array}$ & 7.8 & 22.4 & 20 & 8.74 \\
\hline $\begin{array}{l}\text { Supraspinal ligament } \\
\text { (SSL) }\end{array}$ & 8 & 10.5 & 22 & 3.82 \\
\hline Posterior longitudinal ligament (PLL) & 10 & 7 & 12 & 5.83 \\
\hline Intertransverse ligament (ITL) & 10 & 0.6 & 32 & 0.19 \\
\hline Capsular ligament(CL) & 7.5 & 10.5 & 5 & 15.75 \\
\hline Interspinal ligaments (ISL) & 10 & 14.1 & 13 & 10.85 \\
\hline $\begin{array}{l}\text { ligamentum flavum } \\
\text { (LF) }\end{array}$ & 17 & 14.1 & 15 & 15.98 \\
\hline
\end{tabular}


The number of nodes and elements of the model is reflected in the following figure [Table 4] [Fig. 1]. The contact type of facet joint is friction with a friction coefficient of 0.2 . The rest contact types are set to the binding mode. To improve the efficiency and accuracy of the calculation, The type is set to tetrahedral grid, the size of the articular cartilage grid is $0.5 \mathrm{~mm}$, the screw is $1 \mathrm{~mm}$, and the rest of the objects are set to $2 \mathrm{~mm}$.

Table 4

Number of nodes and elements after grid subdivision for various models

\begin{tabular}{|lll|}
\hline Model & Nod & Element \\
\hline intact & 498843 & 304822 \\
\hline SA & 501616 & 304969 \\
\hline UPS & 765881 & 476073 \\
\hline BPS & 730963 & 444357 \\
\hline UPS-CTLFS & 651481 & 394664 \\
\hline CBT & 729405 & 435188 \\
\hline
\end{tabular}

Six models were set up with boundary and loading conditions in the Static model [16, 21]: bilateral alae sacralis fixation in S1, a vertical axial downward preload of $150 \mathrm{~N}$ applied to the upper surface of L3 and a $10 \mathrm{~N}-\mathrm{m}$ moment along the radial direction on the upper surface of $\mathrm{L} 3$ to simulate six different physiological motions: flexion, extension, right and left bending, and right and left axial rotation. The biomechanical stability of the OLIF under different fixations was investigated by analyzing and comparing ROM, CAGE stresses and internal fixation stresses. The boundary condition settings for the intact model of flexion can be seen in Figure 1.

\section{Results}

\subsection{Validation of the model}

After applying similar loads to our model, we compared our ROM results with those of a cadaveric study conducted by Yamamoto [28]et al[Figure 2]. We found that the results consistent with the reported data.

\subsection{Range of Motion of fusion segment [L4-5] [Figure 3]}

The ROM of the surgical model under a combined load of $150 \mathrm{~N}$ and $10 \mathrm{~N}-\mathrm{M}$ is shown in Figure 5. After insertion of the CAGE, the predicted ROM at surgical segment L4-L5 decreased in all motions the intact model. Compared with all surgical models, the SA model had the greatest ROM in all modes of motion; the CBT had the least ROM in extension and flexion. The BPS and UPS-CTFS were similar in most of the motions, particularly in rotation and lateral bending, and both sets of values were the least active models relative to the remaining movement modes. In axial rotation, the CBT group is similar to the UPS in terms 
of activity. In lateral bending, however, the CBT screws were relatively more active Compared with the UPS-CTFS group and the BPS model, but less active than the SA and UPS.

\subsection{Von Mises stress in CAGE [Figure. 4]}

The Cage had the highest stress in the SA model under different motion loading conditions. Compared with all the surgical models, the cage stress in the BPS model is the lowest without taking the left rotation into consideration. In the case of left rotation, the UPS-CTFS cage had the smallest Von Mises stress, but the specific value was close to that of the BPS. The stresses in the CAGE of the CBT were greater than those in the UPS, UPS-CTFS and BPS in rotation and left and right bending. In extension CBT was similar to BPS and UPS-CTFS. In bending, the CAGE stresses in the CBT were greater than those in the BPS but slightly less than those in the UPS-CTFS.

\subsection{Von Mises stress for internal fixation [Figure. 5][Figure.6]}

The CBT internal fixation was subjected to the highest stress in all motions. The UPS-CFTS was second only to the CBT in all motions, and the BPS internal fixation was the least stressed in flexion, axial rotation and left and right bending. In extension, the UPS internal fixation was least stressed. Except UPSCFTS group, the maximum forward bending stress region of other models was mostly on the titanium rod. In contrast, the maximum stress in the UPS-CFTS was located in the mobile area of the facet joint of the facet screw.

\section{Discussion}

With the development of the OLIF and the associated surgical instruments, OLIF is increasingly being used by spine surgeons. In contrast to lateral lumbar interbody fusion (LLIF) and posterior lumbar interbody fusion (PLIF), OLIF accesses the target disc from the window between the major abdominal vessels and the psoas, thus reducing the risk of lumbar plexus injury and paravertebral tissue destruction[13]. The OLIF is characterized by shorter operation time, less bleeding, less postoperative pain, shorter hospital stay and faster postoperative recovery[29].

However, OLIF techniques are limited by the difficulty in removing lateral recess, ligament flava hypertrophy, and hyperostosis of facet joints directly from the lateral corridor. [30, 31] The improvement of symptoms after OLIF is based on the restoration of disc height, the increase of the foraminal area, the correction of coronal balance, and the indirect decompression of the neural elements by the wider implants[6, 32]. Joseph [33] et al. reported a complication rate of $20.2 \%(380 / 1885)$ for nerve injury in TLIF, while Abe[13] et al. reported a complication rate of $1.2 \%(2 / 155)$ for nerve injury in OLIF, suggesting that the complication rate for nerve injury in OLIF is significantly less than in TLIF.

Subsidence depends on multiple factors related to the low quality of bone of patients, the damage to endplates during their preparation, overdistraction, multilevel fusion, small cages, and different types of instruments. Among the above-mentioned factors[34, 35]. many spinal surgeons believe that different types of instruments are one of the important factors for maintaining the stability of the surgical segment 
and reducing cage subsidence[3]. Although OLIF is an effective and increasingly performed treatment, there is still no consensus regarding the ideal instruments. Different fixation methods have been reported in the literature, including CAGE alone, without the implantation of other internal fixation devices such as SA, UPS, and BPS. Relatively little has been reported on CBT with UPS-CTFS,[32] and to our knowledge, few studies have used FE analysis methods to analyze biomechanics stability analysis of OLIF with various fixation options.

Degenerative diseases of the lumbar spine such as LSS mainly occur at the L4-L5 segment, and OLIF is also commonly performed at the L2-L5 segment[6]. At the same time, due to the indirect decompression of OLIF CAGE, we believe that an intact model simulation could be used to achieve the ideal vertebral space size. Therefore, in this study, the L4-L5 segment fusion model was used to analyze the effect of different implants after OLIF surgery. Because OLIF CAGE can raise the height of intervertebral space, expand the size of intervertebral foramen, thus indirectly achieving decompression[37]. Therefore, we believe that the surgery can indirectly restore the intervertebral height. After surgery, the intervertebral height is restored to a reasonable intervertebral height. We used patients with degenerative lumbar spine disease with relatively normal intervertebral height to implant OLIF CAGE and internal fixation device to simulate the postoperative patients with lumbar degenerative diseases.

\subsection{SA group}

Selvon St. Clair [36] et al. performed an experimental biomechanical study of the OLIF after cadaveric specimens, and obtained dynamic and static biomechanical data. They found that the lumbar spine was comparable to normal lumbar spine biomechanical performance standards after OLIF procedure, while ROM decreased by more than $50 \%$, indicating that the fusion segment was stable enough to withstand a high degree of repetitive loading after OLIF procedure. The studies of Shasti Mark [37] et al. showed that compared to the model with internal fixation, the SA model had the greatest ROM in all directions and the greatest CAGE stress which may increase the potential risk of CAGE subsidence. Their studies have also shown that LLIF alone increases the stability of human spinal motion segments in all loading directions and that $30 \%$ of lumbar levels treated with LLIF showed CAGE subsidence on imaging [38]. In addition, when the subsidence of CAGE occurs, the possible clinical symptoms are axial pain and neurological symptoms, which may be due to loss of indirect decompression space, collapse of the bone structure around the intervertebral body or degeneration. Tempel et al [39] included 297 patients undergoing lateral fusion with SA through a retrospective analysis of prospective data. They found that fusion subsidence was a significant predictor of postoperative revision with the Stand-Alone technique, and therefore recommended the implantation of internal fixation in cases with significant risk factors for fusion collapse. Most authors often combine the use of internal fixation devices to maintain the stability of surgical segment, reduce fusion loosening and promote intervertebral fusion[40]. Therefore, in many cases, such as in patients with osteoporosis [41], SA does not ensure its stability and requires adjunctive fixation, such as pedicle screw to distribute the load over the implanted vertebrae and avoid subsidence of the implant. In our experiments the stress of Cage of SA was greater than the rest of the internal fixation for different motion loads, and the likelihood of postoperative CAGE subsidence was greater than 
the rest of internal fixation devices, but the relative ROM of SA was $25.58 \%$ of that of the normal model L4-L5 segment, reducing the relative ROM and limiting the ROM of fusion segment and providing a good fixation effect.

\subsection{BPS group and UPS}

BPS fixation features three column concept of spine stability and is therefore the gold standard for the treatment of degenerative and traumatic spinal disorders and is widely used as a posterior fixation device after OLIF CAGE implantation[42]. In our study, the BPS showed the least ROM, CAGE stress, and screw stress in different motions, which also means that BPS provides the best biomechanical properties for OLIF. However, there are some disadvantages to BPS fixation, including damage to the paravertebral muscles during instrumentation and postoperative muscle atrophy, risk of nerve damage, vascular injury and increased operative time. In view of the relatively large size of the OLIF fusion and to reduce the risks associated with BPS during surgery, some surgeons choose UPS which can provide better stability and at the same time reduce the cost of the procedure to the patient. UPS causes less damage to the paravertebral muscles, less perioperative bleeding and overall lower cost of the implants. In our study, the relative ROM of the UPS was higher than that of the SA group, but ROM of the surgical segments was greater than that of other internal fixation models. The stress on the CAGE in the UPS group was higher than that of other groups, and there was an increased risk of CAGE subsidence. In a clinical trial, Aoki et al[43] analyzed $1 \mathrm{ss} 25$ patients undergoing transforaminal lumbar interbody fusion and found that the incidence of loosening of the CAGE was higher in patients with UPS (8.3\%) than in those with BPS $(2.1 \%)$.

\subsection{The UPS-CTFS group}

Translaminar facet screw fixation was developed by Magerl in 1984 and is used clinically in patients with acute spinal trauma and degenerative spinal disease [44]. Biomechanical studies have shown that the Translaminar facet screw fixation technique provides a similar degree of spinal stability compared to conventional BPS [45]. Several studies have also demonstrated the biomechanical advantages of transforaminal pedicle screw fixation in terms of reducing flexion, extension and rotation[34, 46, 47]. Biomechanical and clinical studies have shown that UPS-CTFS can achieve similar stability and fusion rates as BPS[48-50]. In addition, the OLIF is long, and wide and can be adjusted, and the CAGE can pass through the entire vertebral body and be fixed in the endplate with relative stability on the bone surface. We therefore conjecture that relatively stable internal fixation can also be achieved with UPS-CTFS. In our study, we used the BPS group to compare with the UPS-CTFS group. The UPS-CTFS group had less ROM value than the BPS group in extension, left and right rotation and left and right bending; in terms of stress on the CAGE, the UPS-CTFS group had more stress than the BPS group in flexion, extension and left and right bending, but the two groups were similar in rotation. The Von Mises stress of the internal fixation device was higher in the UPS-CTFS group compared to the BPS group, with better s stress shielding Therefore, we conclude that UPS-CTFS can achieve similar fixation results as pedicle screws in OLIF and reduce operative time. It is worthwhile to put it into clinical use. In addition, the BPS is technically more complex and more invasive, requiring four pedicle screws and two titanium rods in a single-segment procedure, and causes damage to both lumbar muscles, which can be further reduced when combined 
with percutaneous pedicle screw fixation. However, the pedicle screw is more expensive than the facet screw. Therefore, considering biomechanical stability, technical difficulty and cost, UPS-CTFS may be an alternative to the OLIF BPS and UPS procedures

\subsection{CBT group}

For CBT technique, Santoni[51] proposed in 2009 an inferior-inferior to superior-inferior approach to nailing, a new approach to nailing by increasing the contact between the screw and the cortical bone. In contrast to the pedicle screw technique, this technique requires the screw to be inserted caudally to cephalad in the sagittal plane and medially to laterally in the coronal plane, thus increasing cortical bone contact and maximizing tricortical fixation and increasing the holding screw strength. The article also points out that although the CBT screw is more resistant to axial extraction but less resistant to bending and rotation than the BPS. The use of CBT in osteoporotic patients has a better effect on the resistance to pullout force and can better prevent the occurrence of loosening of the internal fixation. In our study, we found that compared to the BPS, the ROM of the fusion segment was relatively low for the CBT screws in flexion and extension, while in flexion and rotation the ROM was greater than in the UPS-CFTS group, and the stress on the CAGE was greater in the CBT group than in the The BPS group. Although the CBT group had the highest stresses in all types of kinematic loads compared to the other models, they were still less than the yield strength of titanium of 897-1034 Mpa[52]. From the above experiments, in terms of biomechanics, we believe that CBT screws can be an alternative to BPS, especially for patients with osteoporosis. However, the clinical use of this procedure still needs a long-term retrospective study for comprehensive analysis.

\subsection{Limitations}

Our study is based on FE analysis and has several limitations. Firstly, the FE analysis was 3D modelled for CT data. We only selected the skeletal model, the rest models were made by later processing and no tissue model such as muscle skin was included, resulting in some experimental errors. Secondly, we only used the skeletal data of a single person for modelling and computing, ignoring the differences between different people. The description of provertebral disease was not taken into account in the experiment, so the experiment still requires to be validated by long-term clinical trials with large samples and cadaveric experiments.

\section{Conclusion}

The BPS model provides the best biomechanical stability for OLIF, while the SA model has a relatively high risk of postoperative collapse. UPS can be an alternative treatment option to internal fixation due to its ability to well limit the movement of the fusion segment, reduce the Von Mises stress of CAGE, and be less invasive and less expensive to perform. Stresses are relatively high in the UPS-CTFS group compared to the BPS group, and there is a possibility of loosening of the internal fixation in the postoperative period. UPS-CTFS and BPS have less ROM for the surgical segments, thus leading to better stability. The CBT screws, due to their relatively special screw paths, enable them to obtain a better resistance to 
pullout force and also limit the ROM of the surgical segment. But in this experiment, the CBT group was subjected to a greater stress compared to the other groups, and there may be a possibility of broken screws and rods in the postoperative period. In this experiment, the CBT group and the UPS-CTFS group may be a better alternative to BPS, but a comprehensive and long-term evaluation is required in the clinical trials.

\section{Abbreviations}

a stand-alone model (SA), a unilateral pedicle screw model (UPS), a unilateral pedicle screw contralateral translaminar facet screw model (UPS-CTLFS), a bilateral pedicle screw model (BPS) , a cortical bone trajectory screw model (CBT), oblique lumbar interbody fusion (OLIF), Finite Element Analysis (FEA),CT Computed Tomography,dicom (Digital Imaging and Communications in Medicine format)

\section{Declarations}

\section{Ethics approval and consent to participate}

The present study was approved by the Ethics Committee of the Affiliated Hospital of Chengde Medical College. Informed consent obtained from each participant was written. All protocols are carried out in accordance with relevant guidelines and regulations.

\section{Consent for publication}

Not applicable.

\section{Availability of data and materials}

The datasets used and/or analyzed during the current study are available from the corresponding author on reasonable request. Readers can access the data and material supporting the conclusions of the study by contacting Shuyi Zhang at915368073@qq.com.

\section{Competing interests}

The authors declare that they have no conflict of interest.

\section{Funding}

Not applicable.

\section{Authors' contributions}

ZYL designed the study. ZSY and LZP conduct the experiment,ZSY wrote the main manuscript text. LZP and LCS revised manuscript. WYH , FC and ZL analyzed the data. 
Acknowledgements

We would like to thank all the staff in Department of Spine Surgery, Affiliated Hospital of Chengde Medical College for their contribution on our research.

\section{References}

1. The classic. Transplantation of a portion of the tibia into the spine for Pott's disease. A preliminary report. Jama, 57: 885, 1911. Clin Orthop Relat R. 1972; 87:5-8.

2. The classic: the original paper appeared in the New York Medical Journal 93:1013, 1911. I. An operation for progressive spinal deformities: a preliminary report of three cases from the service of the orthopaedic hospital. Clin Orthop Relat Res. 1964; 35:4-8.

3. Xu DS, Walker CT, Godzik J, Turner JD, Smith W, Uribe JS. Minimally invasive anterior, lateral, and oblique lumbar interbody fusion: a literature review. Annals of Translational Medicine. 2018; 6(6):104.

4. Mayer MH. A New Microsurgical Technique for Minimally Invasive Anterior Lumbar Interbody Fusion. Spine. 1997; 22(6):691-699.

5. Silvestre C, Mac-Thiong J, Hilmi R, Roussouly P. Complications and Morbidities of Mini-open Anterior Retroperitoneal Lumbar Interbody Fusion: Oblique Lumbar Interbody Fusion in 179 Patients. Asian Spine Journal. 2012; 6(2):89.

6. Woods KRM, Billys JB, Hynes RA. Technical description of oblique lateral interbody fusion at L1-L5 (OLIF25) and at L5-S1 (OLIF51) and evaluation of complication and fusion rates. The Spine Journal. 2017; 17(4):545-553.

7. Blizzard DJ, Thomas JA. MIS Single-position Lateral and Oblique Lateral Lumbar Interbody Fusion and Bilateral Pedicle Screw Fixation: Feasibility and Perioperative Results. Spine (Phila Pa 1976). 2018; 43(6):440-446.

8. Mehren C, Mayer MH, Zandanell C, Siepe CJ, Korge A. The Oblique Anterolateral Approach to the Lumbar Spine Provides Access to the Lumbar Spine With Few Early Complications. Clinical Orthopaedics \& Related Research. 2016; 474(9):2020-2027.

9. $\mathrm{Kr} \mathrm{W}, \mathrm{Jb} \mathrm{B}, \mathrm{Ra} \mathrm{H}$. Technical description of oblique lateral interbody fusion at L1-L5 (OLIF25) and at L5-S1 (OLIF51) and evaluation of complication and fusion rates. The spine journal: official journal of the North American Spine Society. 2017; 17(4):545-553.

10. Li JXJ, Phan K, Mobbs R. Oblique Lumbar Interbody Fusion: Technical Aspects, Operative Outcomes, and Complications. World Neurosurg. 2017; 98:113-123.

11. Chung N, Jeon $\mathrm{C}$, Lee H, Kweon H. Preoperative evaluation of left common iliac vein in oblique lateral interbody fusion at L5-S1. Eur Spine J. 2017; 26(11):2797-2803.

12. Jin J, Ryu K, Hur J, Seong J, Kim J, Cho H. Comparative Study of the Difference of Perioperative Complication and Radiologic Results. Clinical Spine Surgery: A Spine Publication. 2018; 31(1):3136. 
13. Abe K, Orita S, Mannoji C, Motegi H, Aramomi M, Ishikawa T, Kotani T, Akazawa T, Morinaga T, Fujiyoshi T, et al. Perioperative Complications in 155 Patients Who Underwent Oblique Lateral Interbody Fusion Surgery. Spine. 2017; 42(1):55-62.

14. Zhu G, Hao Y, Yu L, Cai Y, Yang X. Comparing stand-alone oblique lumbar interbody fusion with posterior lumbar interbody fusion for revision of rostral adjacent segment disease. Medicine. 2018; 97(40):e12680.

15. Lu T, Lu Y. Comparison of Biomechanical Performance Among Posterolateral Fusion and Transforaminal, Extreme, and Oblique Lumbar Interbody Fusion: A Finite Element Analysis. World Neurosurg. 2019; 129:e890-e899.

16. H X, W J, N X, X Z, X Z, L Z, X Q, F W, W W, F J. Biomechanical comparison of transforaminal lumbar interbody fusion with 1 or 2 cages by finite-element analysis. Neurosurgery. 2013; 73:s198-s205, s205.

17. Simmons, Edward D. Surgical treatment of patients with lumbar spinal stenosis with associated scoliosis. Clinical Orthopaedics \& Related Research. 2001; 384(384):45-53.

18. Hong, Liu, And, Hirokazu, Ishihara, And, Masahiko, Kanamori, And, Yoshiharu. Characteristics of nerve root compression caused by degenerative lumbar spinal stenosis with scoliosis ScienceDirect. The Spine Journal. 2003; 3(6):524-529.

19. Ruberte LM, Natarajan RN, Andersson GB. Influence of single-level lumbar degenerative disc disease on the behavior of the adjacent segments--a finite element model study. J Biomech. 2009; 42(3):341-348.

20. Shim CS, Park SW, Lee S, Lim TJ, Chun K, Kim DH. Biomechanical Evaluation of an Interspinous Stabilizing Device, Locker. Spine. 2008; 33(22):E820-E827.

21. Mt R, Pm R, Bse, I A, Ag N, V S, Sw C, Bp K, Nr C. Biomechanical evaluation of lateral lumbar interbody fusion with secondary augmentation. Journal of neurosurgery. Spine. 2016; 25(6):720-726.

22. Xu H, Ju W, Xu N, Zhang X, Zhu X, Zhu LF, Qian X, Wen F, Wu W, Jiang F. Biomechanical comparison of transforaminal lumbar interbody fusion with 1 or 2 cages by finite-element analysis. Neurosurgery. 2013; 73:198-205.

23. Hussain M, Nassr A, Natarajan RN, An HS, Andersson GBJ. Relationship between biomechanical changes at adjacent segments and number of fused bone grafts in multilevel cervical fusions: a finite element investigation. Journal of Neurosurgery: Spine. 2014; 20(1):22-29.

24. Wang T, Zhao Y, Cai Z, Wang W, Xia Y, Zheng G, Liang Y, Wang Y. Effect of osteoporosis on internal fixation after spinal osteotomy: A finite element analysis. Clin Biomech. 2019; 69:178-183.

25. V K G, W K, J S H, J N W, L G G. A combined finite element and optimization investigation of lumbar spine mechanics with and without muscles. Spine. 1993; 18(11).

26. Jones AC, Wilcox RK. Finite element analysis of the spine: Towards a framework of verification, validation and sensitivity analysis. Med Eng Phys. 2008; 30(10):1287-1304.

27. Fagan MJ, Julian S, Mohsen AM. Finite element analysis in spine research. Proceedings of the Institution of Mechanical Engineers. Part H, Journal of engineering in medicine. 2002; 216(5):281- 
298.

28. Yamamoto I, Panjabi MM, Crisco T, Oxland T. Three-Dimensional Movements of the Whole Lumbar Spine and Lumbosacral Joint. Spine. 1989; 14(11):1256-1260.

29. Kim JS, Seong JH. Endoscope-assisted oblique lumbar interbody fusion for the treatment of cauda equina syndrome: a technical note. Eur Spine J. 2016; 26(2):1-7.

30. Zairi F, Sunna TP, Westwick HJ, Weil AG, Wang Z, Boubez G, Shedid D. Mini-open oblique lumbar interbody fusion (OLIF) approach for multi-level discectomy and fusion involving L5-S1: Preliminary experience. Orthopaedics \& Traumatology: Surgery \& Research. 2017; 103(2):295-299.

31. Liu C, Wang J, Zhou Y. Perioperative complications associated with minimally invasive surgery of oblique lumbar interbody fusions for degenerative lumbar diseases in 113 patients. Clin Neurol Neurosur. 2019; 184:105381.

32. Patel R, Suh S, Kang S, Nam K, Siddiqui S, Chang D, Yang J. The radiologic and clinical outcomes of oblique lateral interbody fusion for correction of adult degenerative lumbar deformity. Indian J Orthop. 2019; 53(4):502.

33. Joseph JR, Smith BW, Marca FL, Park P. Comparison of complication rates of minimally invasive transforaminal lumbar interbody fusion and lateral lumbar interbody fusion: a systematic review of the literature. Neurosurg Focus. 2015; 39(4):E4.

34. Cao Y, Liu F, Wan S, Liang Y, Jiang C, Feng Z, Jiang X, Chen Z. Biomechanical evaluation of different surgical procedures in single-level transforaminal lumbar interbody fusion in vitro. Clin Biomech. 2017; 49:91-95.

35. Quillo-Olvera J, Lin G, Jo H, Kim J. Complications on minimally invasive oblique lumbar interbody fusion at L2-L5 levels: a review of the literature and surgical strategies. Annals of Translational Medicine. 2018; 6(6):101.

36. St CS, Tan JS, Lieberman I. Oblique lumbar interbody fixation: a biomechanical study in human spines. J Spinal Disord Tech. 2012; 25(4):183-189.

37. Shasti M, Koenig SJ, Nash AB, Bahrami S, Jauregui JJ, O'Hara NN, Jazini E, Gelb DE, Ludwig SC. Biomechanical evaluation of lumbar lateral interbody fusion for the treatment of adjacent segment disease. The Spine Journal. 2019; 19(3):545-551.

38. Marchi L, Abdala N, Oliveira L, Amaral R, Coutinho E, Pimenta L. Radiographic and clinical evaluation of cage subsidence after stand-alone lateral interbody fusion. Journal of Neurosurgery: Spine. 2013; 19(1):110-118.

39. Tempel ZJ, Mcdowell MM, Panczykowski DM, Gandhoke GS, Hamilton DK, Okonkwo DO, Kanter AS. Graft subsidence as a predictor of revision surgery following stand-alone lateral lumbar interbody fusion. J Neurosurg Spine. 2017:1-7.

40. Pham MH, Jakoi AM, Hsieh PC. Minimally invasive L5-S1 oblique lumbar interbody fusion with anterior plate. Neurosurg Focus. 2016; 41 Video Suppl 1(VideoSuppl1):1.

41. Tempel ZJ, Gandhoke GS, Okonkwo DO, Kanter AS. Impaired bone mineral density as a predictor of graft subsidence following minimally invasive transpsoas lateral lumbar interbody fusion. Eur Spine 
J. 2015; 24(S3):414-419.

42. Guo H, Tang Y, Guo D, Zhang S, Li Y, Mo G, Luo P, Zhou T, Ma Y, Liang D, et al. The cement leakage in cement-augmented pedicle screw instrumentation in degenerative lumbosacral diseases: a retrospective analysis of 202 cases and 950 augmented pedicle screws. Eur Spine J. 2019; 28(7):1661-1669.

43. Aoki Y, Yamagata M, Nakajima F, Ikeda Y, Shimizu K, Yoshihara M, Iwasaki J, Toyone T, Nakagawa K, Nakajima A, et al. Examining risk factors for posterior migration of fusion cages following transforaminal lumbar interbody fusion: a possible limitation of unilateral pedicle screw fixation. Journal of Neurosurgery: Spine. 2010;13(3):381-387.

44. Fp M. Stabilization of the lower thoracic and lumbar spine with external skeletal fixation. Clin Orthop Relat R. 1984(189):125-141.

45. Kim S, Lim TJ, Paterno J, Kim DH. A biomechanical comparison of supplementary posterior translaminar facet and transfacetopedicular screw fixation after anterior lumbar interbody fusion. Journal of Neurosurgery: Spine. 2004; 1(1):101-107.

46. Hu Y, Zhu BK, Yuan ZS, Dong WX, Sun XY, Xu JZ, Chen XG. Anatomic study of the lumbar lamina for safe and effective placement of lumbar translaminar facet screws. J Int Med Res. 2019; 47(10):5082-5093.

47. Guo H, Tang Y, Guo D, Luo P, Li Y, Mo G, Ma Y, Peng J, Liang D, Zhang S. Stability Evaluation of Oblique Lumbar Interbody Fusion Constructs with Various Fixation Options: A Finite Element Analysis Based on Three-Dimensional Scanning Models. World Neurosurg. 2020; 138:e530-e538.

48. Cao Y, Zhang W, Liang Y, Feng Z, Jiang C, Chen Z, Jiang X. Translaminar facet joint screw insertion with a rapid prototyping guide template: a cadaver study. Comput Assist Surg. 2019; 24(1):1-6.

49. Cao Y, Liu F, Wan S, Liang Y, Jiang C, Feng Z, Jiang X, Chen Z. Biomechanical evaluation of different surgical procedures in single-level transforaminal lumbar interbody fusion in vitro. Clin Biomech (Bristol, Avon). 2017; 49:91-95.

50. Zeng Z, Zhang J, Song Y, Yan W, Wu P, Tang H, Han J. Combination of Percutaneous Unilateral Translaminar Facet Screw Fixation and Interbody Fusion for Treatment of Lower Lumbar Vertebra Diseases: a Follow-Up Study. Orthop Surg. 2014; 6(2):110-117.

51. Santoni, B. G, Hynes, R. A, Mcgilvray, K. C, Rodriguez-Canessa, G., Lyons, A. S. Cortical bone trajectory for lumbar pedicle screws. Spine J. 2009.

52. Song C, Chang H, Zhang D, Zhang Y, Shi M, Meng X. Biomechanical Evaluation of Oblique Lumbar Interbody Fusion with Various Fixation Options: A Finite Element Analysis. Orthop Surg. 2021; 13(2):517-529.

\section{Figures}



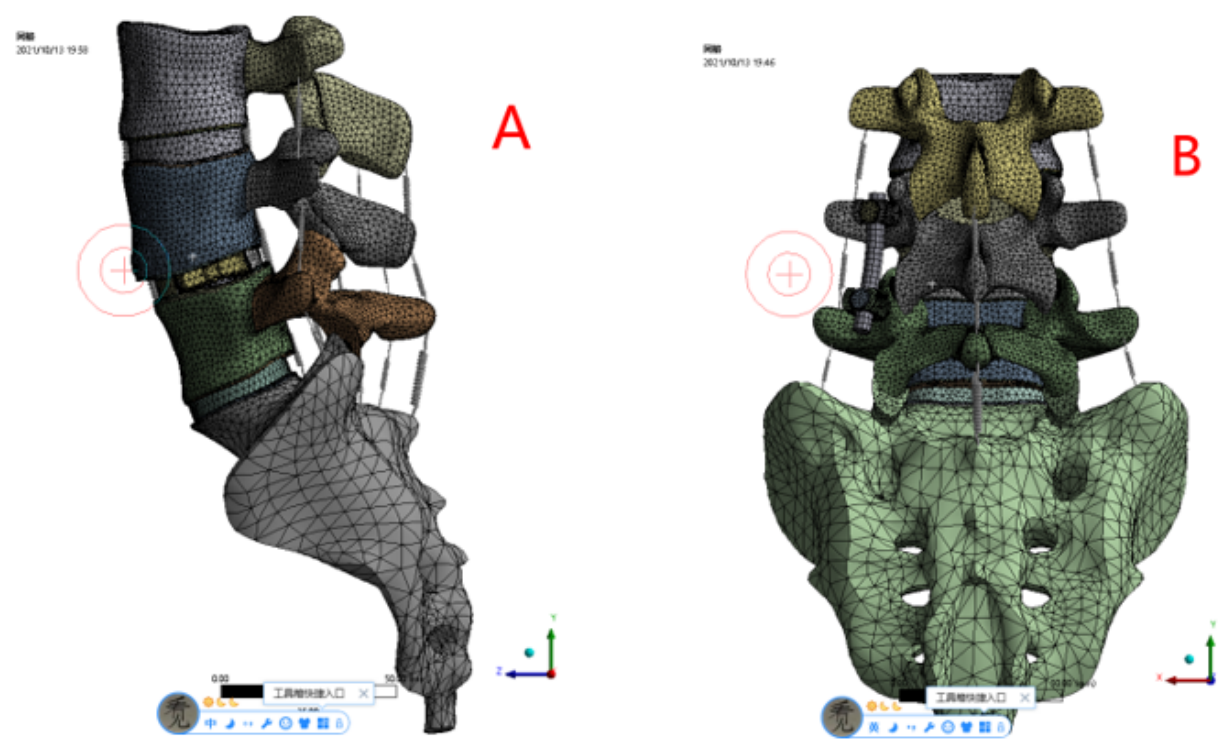

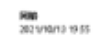
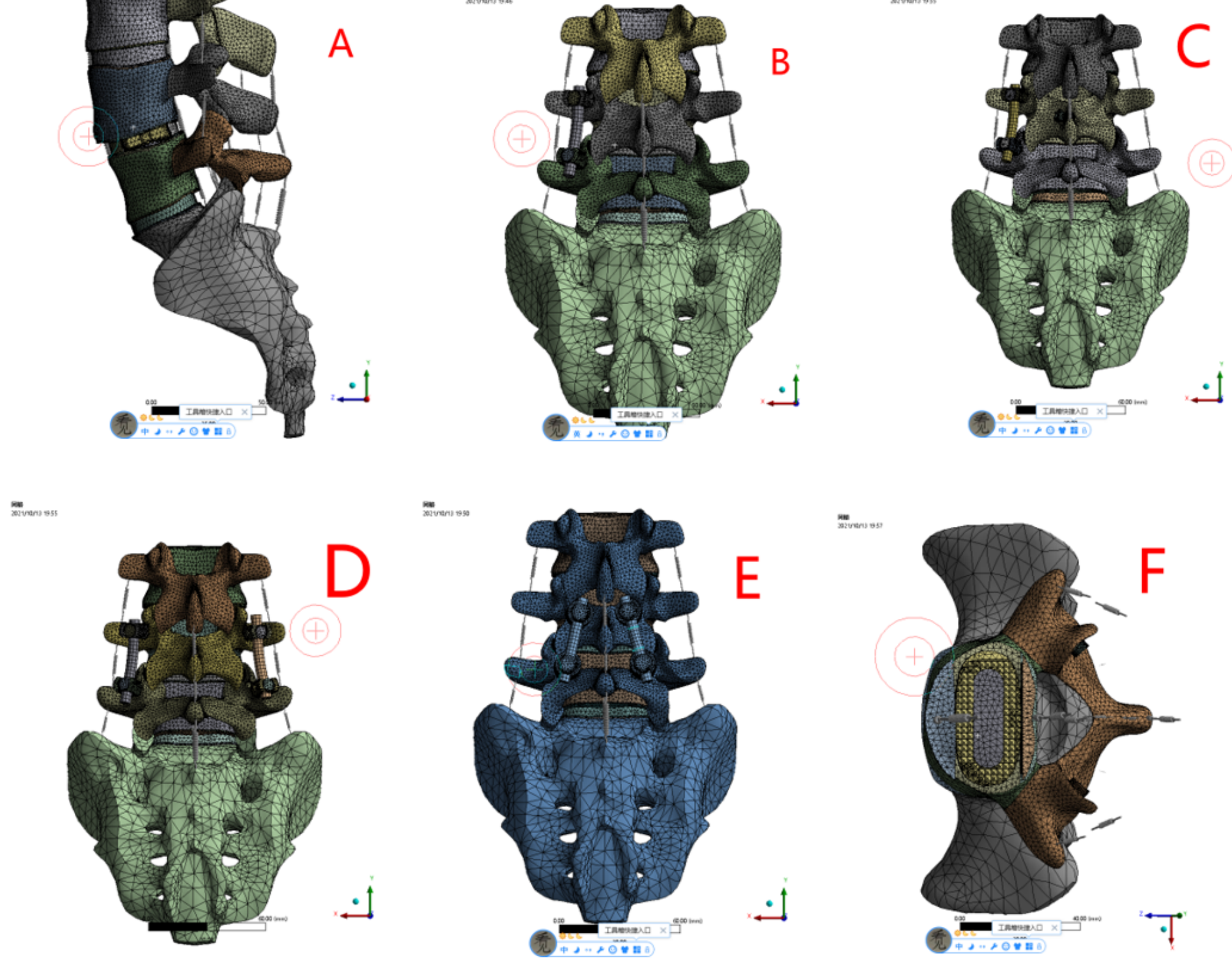

Figure 1

Model mesh division (A)SA model (B)UPS (C)UPS-CFTS (D)BPS (E)CBT (F) The fusion cage in horizontal position 


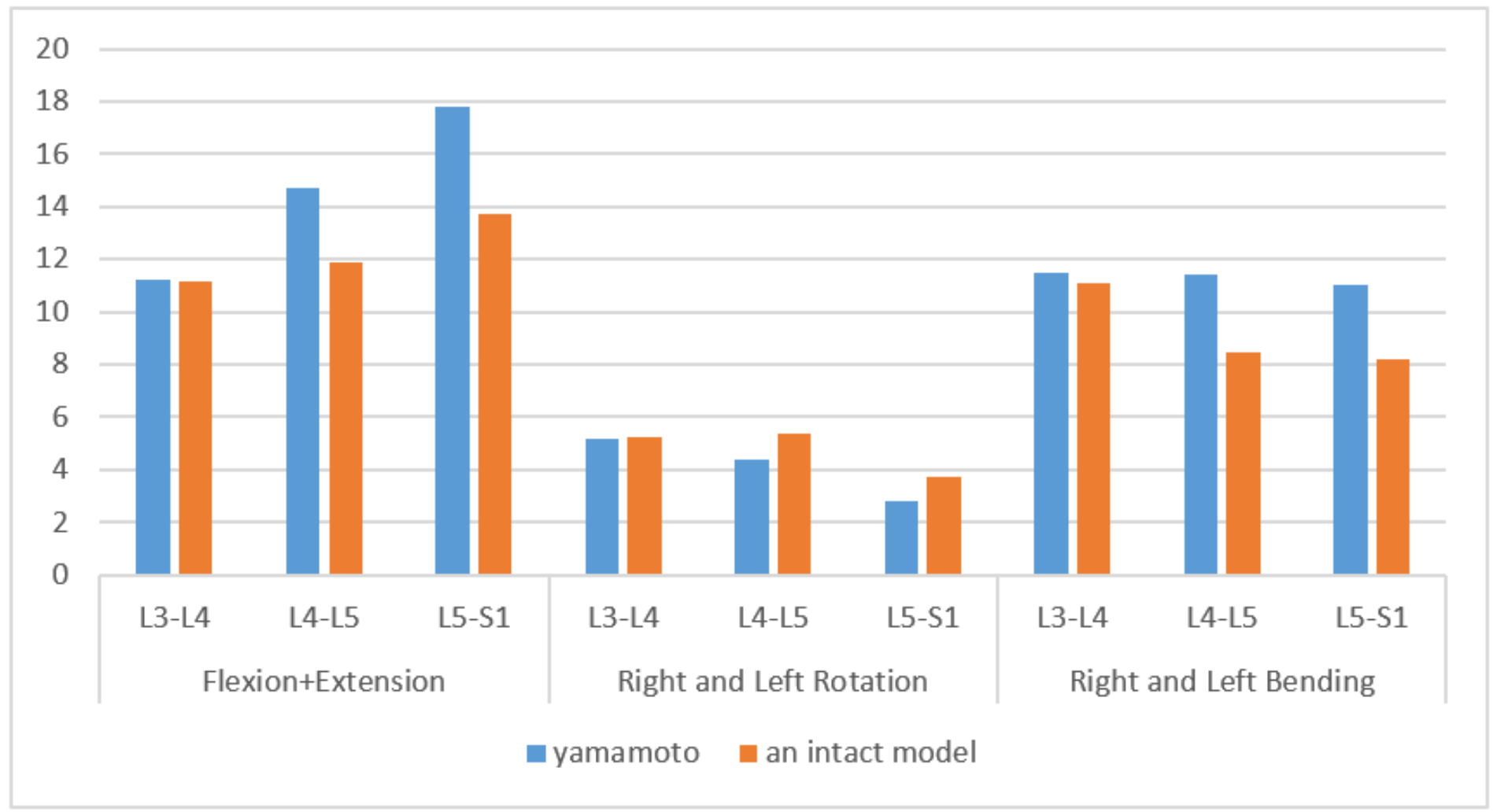

Figure 2

Comparison of range of motion between the intact model and Yamamoto

2

1

0

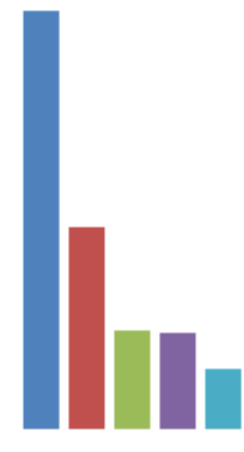

Flexion

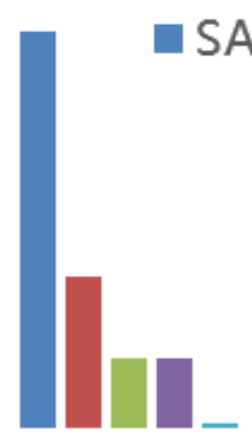

Extension
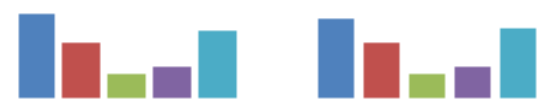

L-Rotation
UPS-CTFS

BPS

CBT
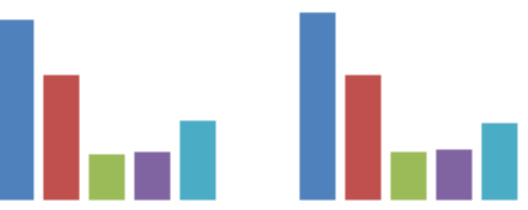

R-Rotation
L-Bending
R-Bending

\section{Figure 3}

ROM of the fusion segments [L4-L5]. 


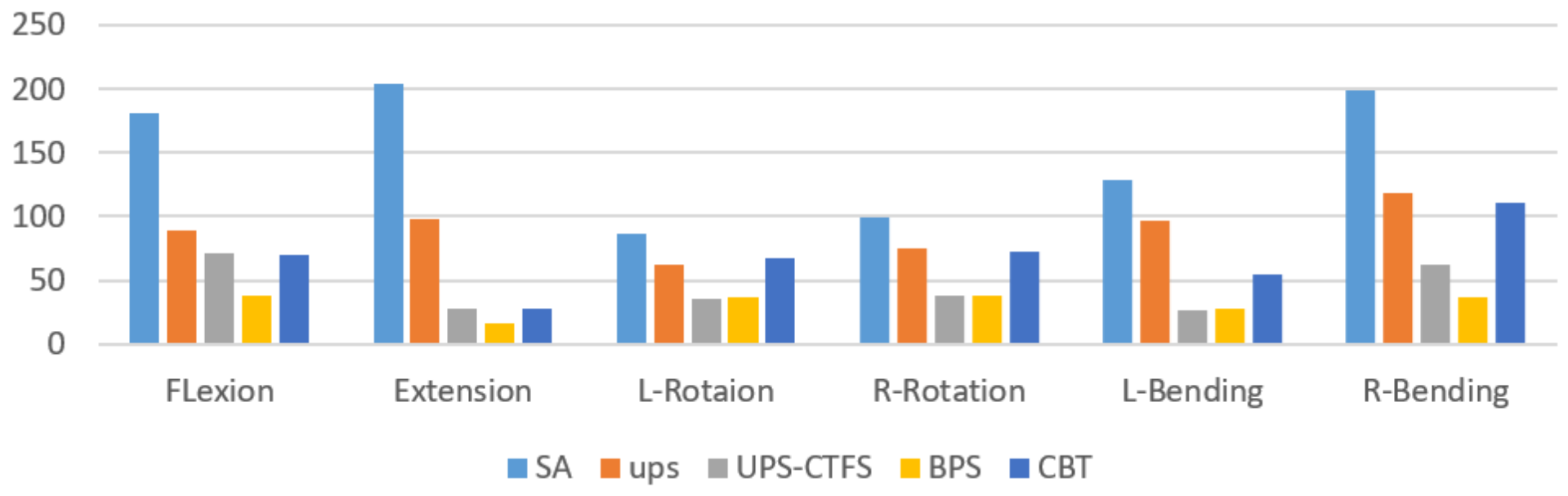

Figure 4

Stress on cage (MPa)

250

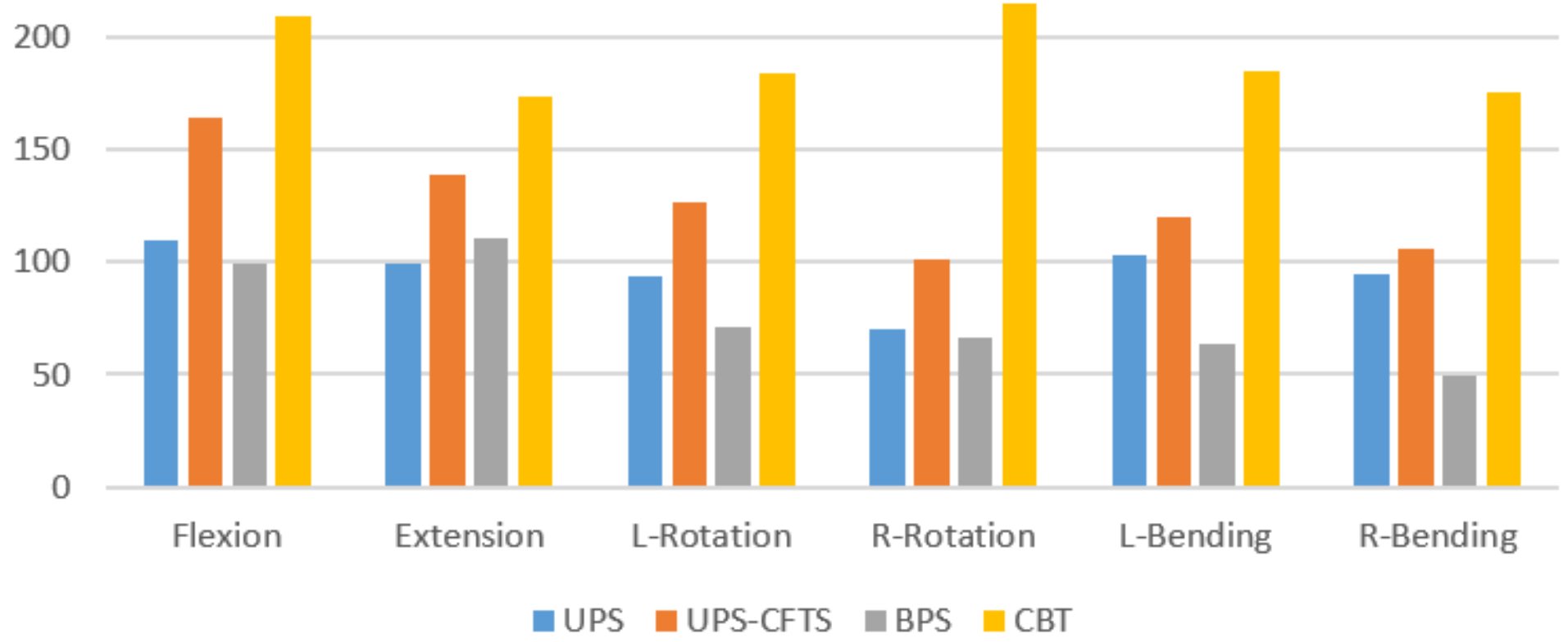

Figure 5

Stress of internal fixation devive (MPa区

展

Figure 6

Von Mises stress of internal fixation during flexion in each group A. a unilateral pedicle screw model \UPS $\triangle$ B. a unilateral pedicle screw contralateral translaminar facet screw model (UPS-CTLFS). C. a bilateral pedicle screw model (BPS) D. a cortical bone trajectory screw model $\triangle \mathrm{CBT} \rrbracket$ 